\title{
Short-lasting unilateral neuralgiform headache attacks with conjunctival injection and tearing syndrome secondary to an epidermoid tumor in the cerebellopontine angle
}

\author{
Case report
}

\author{
Shaun D. Rodgers, M.D., Bryan J. Marascalchi, B.S., Russell G. Strom, M.D., \\ and Paul P. Huang, M.D.
}

Department of Neurosurgery, New York University Langone Medical Center, New York, New York

\begin{abstract}
Short-lasting unilateral neuralgiform headache attacks with conjunctival injection and tearing (SUNCT) syndrome is classified under trigeminal autonomic cephalalgias. This rare headache syndrome is infrequently associated with secondary pathologies. In this paper the authors report on a patient with paroxysmal left retroorbital pain with associated autonomic symptoms of ipsilateral conjunctival injection and lacrimation, suggestive of SUNCT syndrome. After failed medical treatment an MRI sequence was obtained in this patient, demonstrating an epidermoid tumor in the left cerebellopontine angle. The patient's symptoms completely resolved after a gross-total resection of the tumor. This case demonstrates the effectiveness of resection as definitive treatment for SUNCT syndrome associated with tumoral compression of the trigeminal nerve. Early MRI studies should be considered in all patients with SUNCT, especially those with atypical signs and symptoms.

(http://thejns.org/doi/abs/10.3171/2013.1.FOCUS12233)
\end{abstract}

\begin{abstract}
KEY WORDS • epidermoid tumor • cerebellopontine angle • surgery • treatment options - short-lasting unilateral neuralgiform headache attacks with conjunctival injection and tearing syndrome
\end{abstract}

$\mathrm{S}$ HORT-LASTING unilateral neuralgiform headache attacks with conjunctival injection and tearing syndrome is a rare headache disorder classified under TAC. It is characterized by short-lasting unilateral attacks of severe neuralgiform pain associated with prominent lacrimation and redness of the ipsilateral eye. Other autonomic symptoms include nasal congestion, rhinorrhea, and eyelid edema. ${ }^{1}$ Although SUNCT syndrome is a primary headache disorder, it can be associated with secondary pathologies. ${ }^{1,7,14}$ We report on a patient with paroxysmal left retroorbital pain with associated autonomic symptoms of ipsilateral conjunctival injection and lacrimation, suggestive of SUNCT, secondary to an epidermoid tumor in the left CPA. The symptoms completely resolved after a retrosigmoid craniotomy and resection of the tumor.

Abbreviations used in this paper: $\mathrm{CPA}=$ cerebellopontine angle; $\mathrm{DW}=$ diffusion-weighted; SUNCT = short-lasting unilateral neuralgiform headache attacks with conjunctival injection and tearing; $\mathrm{TAC}=$ trigeminal autonomic cephalalgia.

\section{Case Report}

History and Examination. This 33-year-old righthanded man presented with 6-8 months of left retroorbital headaches with extension into the mandible. The intensity of the pain was graded at 10 of 10 according to the visual analog scale, and he described the quality of pain as either "burning" or "shooting." His headaches occurred at 8-minute intervals throughout the day. The attacks would last approximately 30-60 seconds. Symptoms associated with the attacks included conjunctival injection, lacrimation, and nasal congestion. Photophobia and phonophobia were noted during the attacks. In addition, he complained of difficulty swallowing and leftsided blurry vision during the episodes. Jaw opening, chewing, and head movement triggered his symptoms.

The patient presented numerous times to the emergency room and to the clinic, where he was unsuccessfully treated with multiple medications including gabapentin, duloxetine (Cymbalta), pregabalin (Lyrica), oxcarbapene, phenobarbital, and morphine. Antiinflammatory 
drugs, carbamazepine, and steroids were ineffective. $\mathrm{He}$ also underwent 2 dental extractions without relief of his symptoms. The patient had no medical or family history of headache disorders including migraines. Results of his neurological examination were unremarkable except for a left-sided hypesthesia in the maxillary division of the trigeminal nerve (V2).

Imaging Findings. The patient was referred to our institution after CT imaging showed a left-sided CPA hypodensity with mild mass effect near the pons and exiting cranial nerves. An MRI study revealed a mass next to the left ventrolateral pons that measured approximately 3.1 $\mathrm{cm}$ in the anteroposterior direction. The mass extended from the ambient cistern to the Meckel cave, causing upward displacement of the left trigeminal nerve and the 7th-8th cranial nerve complex. The mass was hypointense to gray matter on T1-weighted, hyperintense on T2-weighted, and heterogeneous on FLAIR sequences. The lesion exhibited restriction on DW imaging, with corresponding dropout on apparent diffusion coefficient mapping. These imaging features were suggestive of an epidermoid tumor (Fig. 1).

Operation. The patient underwent a left retrosigmoid craniotomy for resection of the CPA lesion (Video 1).

VIDEO 1. Clip showing resection of a CPA epidermoid tumor.

Click here to view with Media Player. Click here to view with Quicktime.

The mass was soft and pearly white, consistent with an epidermoid cyst. There was significant compression of the 5th-10th cranial nerves (Fig. 2). The tumor was removed in a piecemeal fashion, and we were able to achieve a gross-total resection.

Postoperative Course. The postoperative MRI showed a gross-total resection with no residual diffusion restriction (Fig. 3). Pathological examination confirmed the diagnosis of an epidermoid tumor. The patient reported complete resolution of his facial pain and no longer experienced any signs or symptoms of SUNCT. Postoperatively he exhibited a mild 6th nerve palsy and numbness of the mandibular division of the trigeminal nerve (V3) on the left, both of which resolved by 2 weeks. The patient's pain medications were gradually discontinued, and he remained symptom free without medications at his 6-month follow-up.
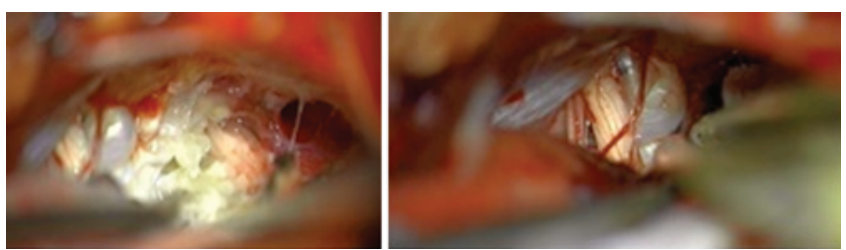

FIG. 2. Intraoperative photographs demonstrating tumor adjacent to the trigeminal nerve and 7th-8th cranial nerve complex. Left: Trigeminal nerve to the right and complex of 7th and 8th cranial nerves to the left. Right: Tumor intimately associated with the 7th-8th nerve complex.

\section{Discussion}

The SUNCT syndrome is considered a subset of short-lasting unilateral neuralgiform headache attacks with cranial autonomic symptoms. The syndrome is classified under the TACs, a group that includes cluster headaches and paroxysmal hemicranias. The TACs are characterized by unilateral pain in the trigeminal nerve distribution that is associated with ipsilateral cranial autonomic symptoms. ${ }^{5}$ The underlying pathophysiology in this group arises from the activation of the trigeminal system with disinhibition of the trigeminofacial reflex. ${ }^{9,10}$ Functional MRI studies show ipsilateral hypothalamic activation, suggesting a relationship with pain-modulating and autonomic systems., ${ }^{8,10}$

Headache disorders are grouped according to pain intensity, response to therapy, severity of autonomic symptoms, attack frequency, and duration of the attacks. ${ }^{3}$ Among the TACs, the SUNCT syndrome has the shortest duration and highest frequency of attacks. The SUNCT syndrome is more common in males, and the mean age at onset (in both sexes) is approximately 50 years. ${ }^{10}$ Attacks last 2-600 seconds, with a mean length of roughly 1 minute. ${ }^{2,5,10}$ Attacks lasting longer than 240 seconds often comprise closely grouped stabs or saw-tooth attacks. ${ }^{2}$ Attack frequency may range from a single episode to hundreds per day. Some patients have so many attacks that they are unable to quantify the actual number. ${ }^{2,10}$

The most common differential diagnosis of SUNCT is trigeminal neuralgia. ${ }^{3}$ Both SUNCT syndrome and trigeminal neuralgia are short-lasting, exhibit cutaneous triggers, and present with lancinating pain associated with autonomic symptoms in the trigeminal distribu-
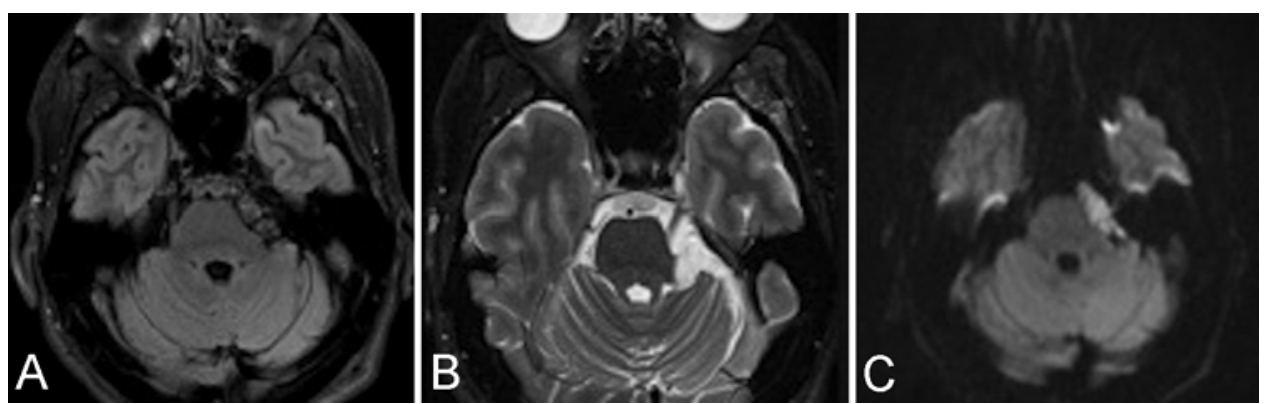

FIG. 1. Preoperative neuroimaging of the epidermoid tumor revealing isointensity on FLAIR (A), hyperintensity on T2-weighted (B), and restricted diffusion on DW (C) imaging. 


\section{Occurrence of SUNCT syndrome secondary to epidermoid tumor}
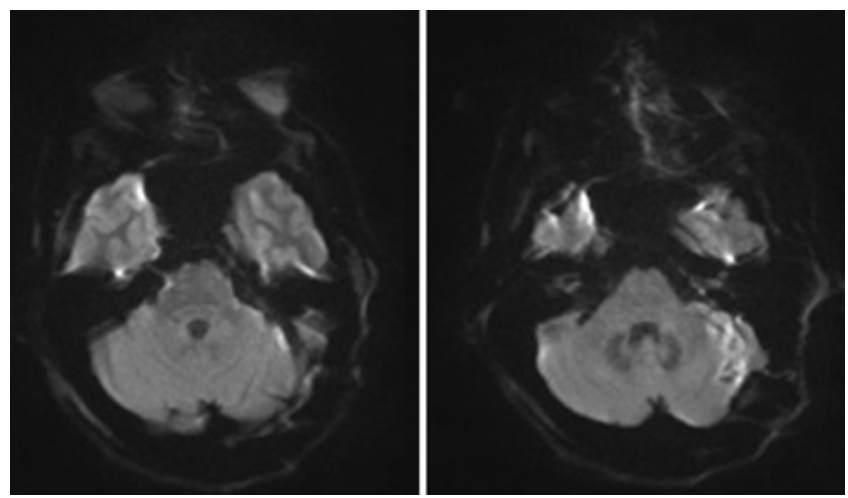

FIG. 3. Postoperative DW imaging studies with no restriction of diffusion, confirming gross-total resection.

tion. ${ }^{2,11}$ However, unlike SUNCT syndrome, trigeminal neuralgia has milder autonomic symptoms and usually responds to carbamazepine. ${ }^{4}$ Our patient fulfilled all the diagnostic criteria for SUNCT (Table 1); experienced significant autonomic symptoms including conjunctival injection, lacrimation, and nasal congestion; and failed to respond to carbamazepine. ${ }^{5}$

The SUNCT syndrome is primarily idiopathic but has been reported in conjunction with a variety of intracranial lesions including pituitary tumors, arteriovenous malformations, cavernous hemangiomas, HIV/AIDS posterior fossa lesions, meningiomas, vascular compression of the trigeminal nerve, and osseous lesions of the posterior fossa. ${ }^{1,2,7,10,12,14}$ Criteria have been proposed for establishing a causal relationship in a patient with a lesion and a headache disorder (Table 2). ${ }^{12}$ In the case reviewed here, all the criteria were met for establishing a causal link between the epidermoid tumor and SUNCT syndrome. To our knowledge this is the first case of SUNCT secondary to an epidermoid tumor in the CPA in which there was resolution of symptoms after a retrosigmoid craniotomy and resection. A case of short-lasting unilateral neuralgiform headache attacks with cranial autonomic symptoms attributed to an epidermoid cyst in the right CPA and treated successfully with surgery was recently reported. ${ }^{6}$ Our case fulfills the full criteria for SUNCT with resolution after resection.

Idiopathic SUNCT syndrome is clinically indistin-

TABLE 1: Classification of SUNCT according to the International Classification of Headache Disorders: 2nd Edition*

\section{A. At least 20 attacks fulfilling criteria B-D \\ B. Attacks of unilateral orbital, supraorbital or temporal stabbing or pulsating pain lasting 5-240 seconds \\ C. Pain is accompanied by ipsilateral conjunctival injection and lacrimation \\ D. Attacks occur with a frequency from 3 to 200 per day \\ E. Not attributed to another disorder}

* Headache Classification Subcommittee of the International Headache Society: The International Classification of Headache Disorders, ed. 2. Cephalalgia 24 (Suppl 1):23-136, copyright @ 2004 by Sage Publications. Reprinted by permission of SAGE.
TABLE 2: Proposed criteria for defining a causal relationship between the associated pathology and the headache syndrome*

1. Close temporal relationship between the associated disease and the onset of pain.

2. Side concordance between the unilateral pain and the lesion, if localized.

3. Surgical remission, if the patient was operated on, or prompt remission after aetiological medical therapy, if indicated, without need of constant indomethacin administration.

4. Prolonged post-treatment follow-up, in order to exclude a relapse of the headache attacks or improvement due to spontaneous remission.

* Trucco M, Mainardi F, Maggioni F, Badino R, Zanchin G: Chronic paroxysmal hemicrania, hemicrania continua and SUNCT syndrome in association with other pathologies: a review. Cephalalgia 24(3):173-184, copyright (C) 2004 by Sage publications. Reprinted by permission of SAGE.

guishable from SUNCT syndrome caused by a structural lesion. Neuroimaging should be considered in all TAC or TAC-like disorders, especially when atypical signs or symptoms are present.13 Our patient presented with trigeminal hypesthesia and dysphagia, both uncommon findings in SUNCT syndrome.

\section{Conclusions}

The SUNCT syndrome is a rare headache disorder that is infrequently associated with secondary intracranial lesions, with few reports of successful neurosurgical intervention. To our knowledge this is the first case of SUNCT secondary to an epidermoid tumor in the CPA in which there was resolution of symptoms after a retrosigmoid craniotomy and resection. This case demonstrates the importance of early MRI studies in the workup and treatment of SUNCT.

\section{Disclosure}

There were no sources of funding in preparation of this manuscript. There are no conflicts of interest in this manuscript.

Author contributions to the study and manuscript preparation include the following. Conception and design: Rodgers, Huang. Acquisition of data: Rodgers, Marascalchi, Strom. Analysis and interpretation of data: all authors. Drafting the article: Rodgers, Marascalchi. Critically revising the article: all authors. Reviewed submitted version of manuscript: all authors. Study supervision: Huang.

\section{References}

1. Adamo MA, Drazin D, Popp AJ: Short-lasting, unilateral neuralgiform headache attacks with conjunctival injection and tearing syndrome treated successfully with transsphenoidal resection of a growth hormone-secreting pituitary adenoma. J Neurosurg 109:123-125, 2008

2. Cohen AS, Matharu MS, Goadsby PJ: Short-lasting unilateral neuralgiform headache attacks with conjunctival injection and tearing (SUNCT) or cranial autonomic features (SUNA) - a prospective clinical study of SUNCT and SUNA. Brain 129:2746-2760, 2006

3. Goadsby PJ, Cittadini E, Burns B, Cohen AS: Trigeminal 
autonomic cephalalgias: diagnostic and therapeutic developments. Curr Opin Neurol 21:323-330, 2008

4. Goadsby PJ, Matharu MS, Boes CJ: SUNCT syndrome or trigeminal neuralgia with lacrimation. Cephalalgia 21:82-83, 2001

5. Headache Classification Subcommittee of the International Headache Society: The international classification of headache disorders, 2nd edition. Cephalalgia 24 (Suppl 1):23136, 2004

6. Jiménez Caballero PE, Portilla Cuenca JC, Casado Naranjo I: Short-lasting unilateral neuralgiform headache attacks with cranial autonomic symptoms (SUNA) secondary to epidermoid cyst in the right cerebellopontine angle successfully treated with surgery. J Headache Pain 12:385-387, 2011

7. Kutschenko A, Liebetanz D: Meningioma causing gabapentinresponsive secondary SUNCT syndrome. J Headache Pain 11:359-361, 2010

8. May A, Bahra A, Büchel C, Turner R, Goadsby PJ: Functional magnetic resonance imaging in spontaneous attacks of SUNCT: short-lasting neuralgiform headache with conjunctival injection and tearing. Ann Neurol 46:791-794, 1999

9. Paliwal VK, Singh P, Kumar A, Rahi SK, Gupta RK: Shortlasting unilateral neuralgiform headache with conjunctival injection and tearing (SUNCT) with preserved refractory period: report of three cases. J Headache Pain 13:167-169, 2012

10. Pareja JA, Cuadrado ML: SUNCT syndrome: an update. Expert Opin Pharmacother 6:591-599, 2005

11. Sesso RM: SUNCT syndrome or trigeminal neuralgia with lacrimation and conjunctival injection? Cephalalgia 21:151153,2001
12. Trucco M, Mainardi F, Maggioni F, Badino R, Zanchin G: Chronic paroxysmal hemicrania, hemicrania continua and SUNCT syndrome in association with other pathologies: a review. Cephalalgia 24:173-184, 2004

13. Wilbrink LA, Ferrari MD, Kruit MC, Haan J: Neuroimaging in trigeminal autonomic cephalgias: when, how, and of what? Curr Opin Neurol 22:247-253, 2009

14. Williams M, Bazina R, Tan L, Rice H, Broadley SA: Microvascular decompression of the trigeminal nerve in the treatment of SUNCT and SUNA. J Neurol Neurosurg Psychiatry 81:992-996, 2010

Manuscript submitted June 21, 2012.

Accepted January 11, 2013.

Please include this information when citing this paper: DOI: 10.3171/2013.1.FOCUS12233.

Supplemental online information:

Video: http://mfile.akamai.com/21490/wmv/digitalwbc.download. akamai.com/21492/wm.digitalsource-na-regional/focus12-233 video.asx (Media Player).

http://mfile.akamai.com/21488/mov/digitalwbc.download.akamai. com/21492/qt.digitalsource-global/focus 12-233_video.mov (Quicktime).

Address correspondence to: Shaun D. Rodgers, M.D., Department of Neurosurgery, New York University Langone Medical Center, 462 First Avenue, Suite 7S4, New York, New York, 10016. email: shaun.rodgers@nyumc.org. 